This is the final peer-reviewed accepted manuscript of:

Luschi A., Di Franco R., Turillazzi B., ladanza E. (2020) System for Monitoring Environmental Parameters in a Hospital Facility. In: Badnjevic A., Škrbić R., Gurbeta Pokvić L. (eds) CMBEBIH 2019. CMBEBIH 2019. IFMBE Proceedings, vol 73. Springer, Cham. https://doi.org/10.1007/978-3-030-17971-7_62

The final published version is available online at:

https://doi.org/10.1007/978-3-030-17971-7_62

Rights / License:

The terms and conditions for the reuse of this version of the manuscript are specified in the publishing policy. For all terms of use and more information see the publisher's website. 


\title{
System for Monitoring Environmental Parameters in a Hospital Facility
}

\author{
Alessio Luschi, Riccardo Di Franco, Beatrice Turillazzi, \\ and Ernesto ladanza
}

\begin{abstract}
The paper presents a system for managing and monitoring environmental parameters in hospital facilities. It relies on existing data collected with a CAFM (Computer Aided Facility Management) system and allows granted users (such as CEO or Energy Manager) to monitor the ongoing energy consumption of a given activity area (Operating Rooms, Diagnostics, Intensive Care Units, Wards) in relation to the compliance to relevant threshold parameters. The system easily outputs aggregated data via PDF reporting.
\end{abstract}

\section{Keywords}

Environment - Energy - Hospital - Managing • CAFM

\section{Introduction}

A typical hospital facility consumes about 2.5 times the energy of an office. In the European Union (EU) there are about 15,000 hospitals which are responsible for the $5 \%$ of the total carbon-dioxide emissions in the whole EU. In this

\footnotetext{
A. Luschi $(\bowtie) \cdot$ E. Iadanza

Department of Information Engineering, University of Florence, Florence, Italy

e-mail: alessio.luschi@unifi.it

E. Iadanza

e-mail: ernesto.iadanza@unifi.it

\section{R. Di Franco}

Bachelor's Degree in Mechanical Engineering, University

of Florence, Florence, Italy

e-mail: riccardo.difranco@stud.unifi.it

B. Turillazzi

Department of Architecture, University of Bologna, Bologna, Italy

e-mail: beatrice.turillazzi@unibo.it
}

context the energy saving associated with a hospital gets a high relevance, and it comes clear that a well designed system to correctly manage the environmental impact of such a premise is crucial. These systems allow not only to minimize the cost of the energy demand, but also to save the amount of carbon-oxide introduced in the atmosphere.

In this scenario it appears clear that a hospital-oriented Computer Aided Facilities Management (CAFM) has to manage and provide performance indicators about the energy consumption and saving, in order to cut the costs for energy supply, giving useful information to the top management as a decision support system.

Thus, the designed application relies on a custom CAFM $[1,2]$ in order to assess and aggregate data which can be previously collected or directly fed in real-time by the system of energy sensors.

\section{Methods}

STREAMER is a research project which aims to halve the energy consumption and the $\mathrm{CO}_{2}$-emissions for new or renewed hospital facilities in Europe in the next 10 years. It is co-financed inside the European Project named "Optimised design methodologies for energy-efficient buildings integrated in the neighborhood energy systems" [3].

The Hospital University Campus of Careggi, in Florence (Italy) is one of the four case studies for the STREAMER Project, so its CAFM system has been re-designed in order to correctly collect and store the energy parameters identified within the project.

First of all, one of the seven STREAMER typologies has been assigned to every single hospital space, to highlight its efficiency:

- Comfort Class

- Hygienic Class

- Equipment Class

- Construction Class 
- User Profile Class

- Access Security Class

- Bouwcollege Layer Class.

Then, by combining the STREAMER typology, the destination of use and the activity area of the spaces, the following five energy classes have been identified:

- Operating Rooms

- Intensive Care Units-Surgery Outpatient ClinicsHigh Speciality Diagnostics (MRI, CT, Angio-CT)Laboratories

- Ward-Low Speciality Diagnostics (radiography, US)

- Outpatient Clinics

- Offices-Teaching Rooms-Alleys-Other.

Each of the above energy classes has its own energy parameters: Winter Temperature, Summer Temperature, Winter Humidity and Summer Humidity.

Moreover, other environmental parameters have also been designed, more focused on compliance to legislative standards (for example Air Ventilation), which are used by external facility management applications within the hospital [4-6].

Each one of these parameters has its own minimum and maximum threshold value as reported in Table 1.

Once the data have been collected and stored inside the hospital's CAFM system [7], the main requirement is to have an efficient managing system in order to update and monitor the parameters, query a single room (or a group of them) or a set of rooms with given attributes (i.e. all the rooms with at least one parameter - or a given parameteroutside the threshold range).

\section{Results}

The designed system is an HTML5 compliant web-application with a standard Javascript, jQuery and Bootstrap client framework which interacts via AJAX calls with RESTful Web APIs coded using Windows. NET 4.5 Framework.

The system analyzes a set of real values for the input parameters and matches them with the theoretical ones associated with the energy class of a given room. These input real values can be fed to the system directly inserting them in the database using a data entry form or creating a JSON file and transferring it through a dedicated REST Web API, using a HttpPost message. This functionality is designed to make the system fully integrable with any third-party sensors management system (SMS). In fact, by simply making the WebAPI accessible (via Intranet of VPN), the SMS could post the properly formatted actual values, implementing a conjunct real-time monitoring system.

The JSON string must have the following format:

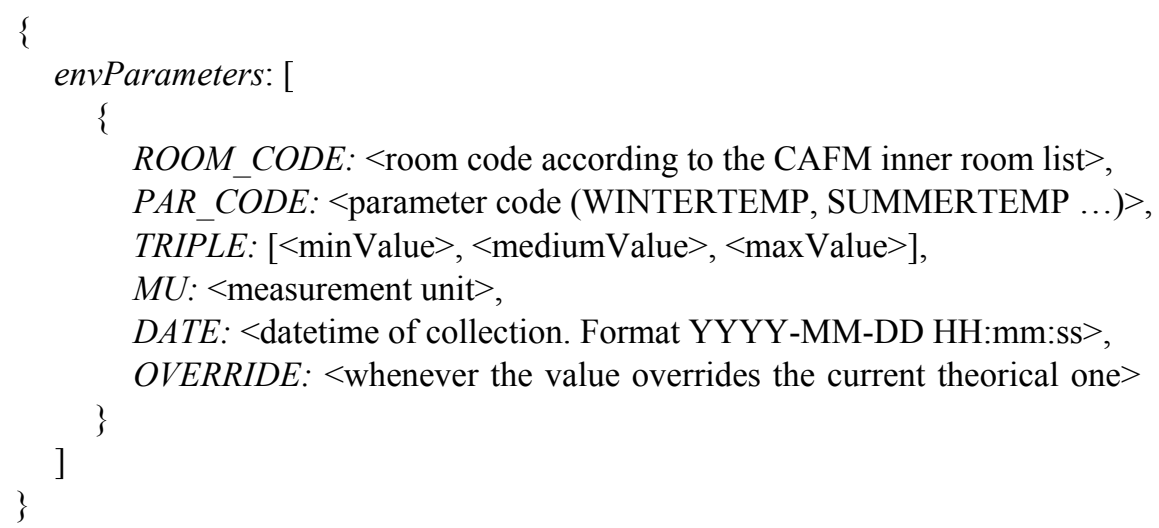

Table 1 Environmental parameters related to the destination of use of the rooms

\begin{tabular}{l|l|l|l|l}
\hline Energy class & $\begin{array}{l}\text { Winter } \\
\text { temperature }\end{array}$ & $\begin{array}{l}\text { Summer } \\
\text { temperature }\end{array}$ & $\begin{array}{l}\text { Winter } \\
\text { humidity }\end{array}$ & $\begin{array}{l}\text { Summer } \\
\text { humidity }\end{array}$ \\
\hline O.R. & $22{ }^{\circ} \mathrm{C} \pm 2{ }^{\circ} \mathrm{C}$ & $22{ }^{\circ} \mathrm{C} \pm 2{ }^{\circ} \mathrm{C}$ & $50 \% \pm 5 \%$ & $50 \% \pm 10 \%$ \\
\hline $\begin{array}{l}\text { ICU/high speciality } \\
\text { diagnostics }\end{array}$ & $21^{\circ} \mathrm{C} \pm 1{ }^{\circ} \mathrm{C}$ & $23{ }^{\circ} \mathrm{C} \pm 1{ }^{\circ} \mathrm{C}$ & $50 \% \pm 10 \%$ & $50 \% \pm 10 \%$ \\
\hline $\begin{array}{l}\text { Ward/low speciality } \\
\text { diagnostics }\end{array}$ & $21^{\circ} \mathrm{C} \pm 1{ }^{\circ} \mathrm{C}$ & $25{ }^{\circ} \mathrm{C} \pm 1{ }^{\circ} \mathrm{C}$ & $50 \% \pm 10 \%$ & $50 \% \pm 10 \%$ \\
\hline Outpatient & $21^{\circ} \mathrm{C} \pm 1{ }^{\circ} \mathrm{C}$ & $25{ }^{\circ} \mathrm{C} \pm 1{ }^{\circ} \mathrm{C}$ & $50 \% \pm 10 \%$ & $50 \% \pm 10 \%$ \\
\hline Office/other & $21^{\circ} \mathrm{C} \pm 1{ }^{\circ} \mathrm{C}$ & $25^{\circ} \mathrm{C} \pm 1{ }^{\circ} \mathrm{C}$ & $50 \% \pm 10 \%$ & $50 \% \pm 10 \%$ \\
\hline
\end{tabular}


Fig. 1 Details section. Winter and summer temperatures together with winter and summer humidity are shown. Current values are displayed on the left column compared with the theoretical ones on the right side

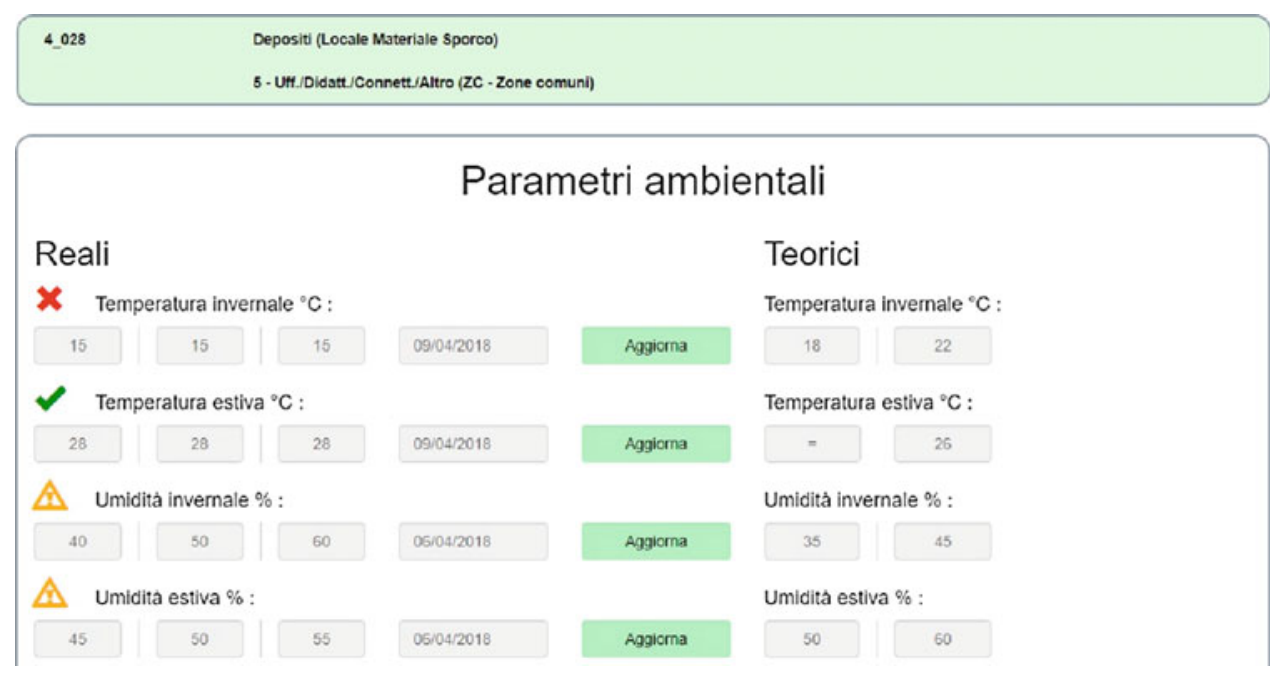

Room_Code,Par_Code and MU are strings, Triple is an array of 3 strings, Date is a datetime object while Override is a Boolean value.

The comparison between the theoretical values and the real ones is performed using a dedicated window (Fig. 1).

In the upper section the system shows the analyzed room code, the Destination of Use of the room together with its Energy Class.

In the main central section, the environmental parameters are shown, divided by typologies. On the left the current values are shown with the date of collection, with the corresponding theoretical on the right.

The "compliant", "not compliant" or "warning" icons represent the degree of compliance of the real value to its theoretical one:

- If the range of values for the collected parameter is fully contained inside the range for the theoretical ones, the icon will be a green tick mark;

- If it is only partially enclosed inside the range for the theoretical parameters, the icon will be a yellow warning sign;

- Otherwise, the icon will be a red alert cross.

By clicking the Update button, a modal window is opened to edit or input new values for the given row. The system allows a user to override an actual value for a given date (not necessarily the last collected one). This function is only available for a granted user, such the Energy Manager or Top Management Office member. User managing is entrusted to the System Administrator, which would add the allowed users to a grant list: the login password is the same of the local Intranet area, managed via LDAP (Lightweight Directory Access Protocol).

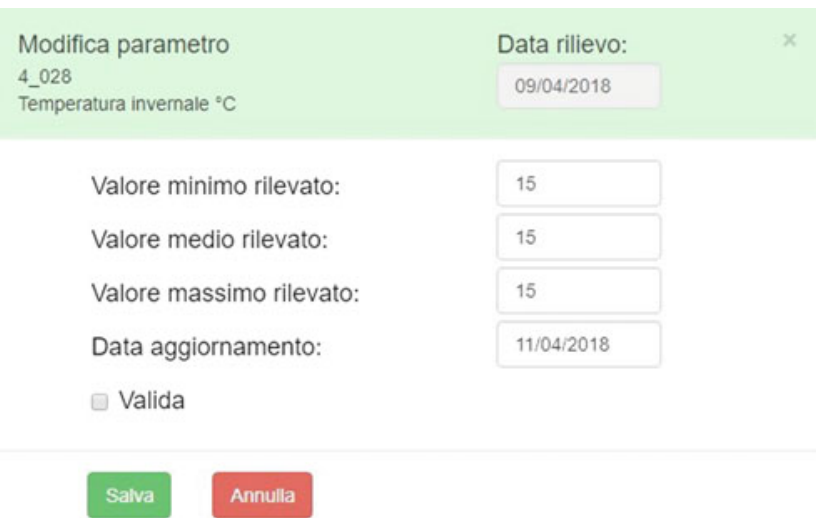

Fig. 2 Modal window with edit and override functionalities. Survey date and specification of the edited parameters are shown in the header. Minimum, maximum and average values are also displayed below together with the update date and a confirm checkbox

A granted user can also override a parameter (or all of them) without editing its value by clicking the Validate checkbox: this makes the real collected valued compliant to the theoretical range even if the values would not be (manual validation functionality) (Fig. 2).

The manual validation functionality allows a granted user to validate a parameter which slightly differ from the theoretical range, but which is assessed to represent a solid and consistent exception for the analyzed room.

The system can also produce a detailed report about the summary of the compliance of a room, a floor, a premise or the whole hospital to the energy parameters. A plot of the time evolution of the minimum, medium and maximum values of each parameter is also available (Fig. 3). 
Fig. 3 a Report for the compliance of rooms grouped by floor and premise. The pie-chart on the left shown the percentage of compliance (green), non-compliance (red) and partially compliance (yellow) for the selected building. Tables for detailed compliance of each room to the analyzed parameters, grouped for floor, are also shown on the right side. $\mathbf{b}$ Report showing the time evolution of minimum (red), medium (blue) and maximum (green) values for each current parameter of a given room. Theorical minimum (orange) and maximum's (yellow) evolution are also plotted
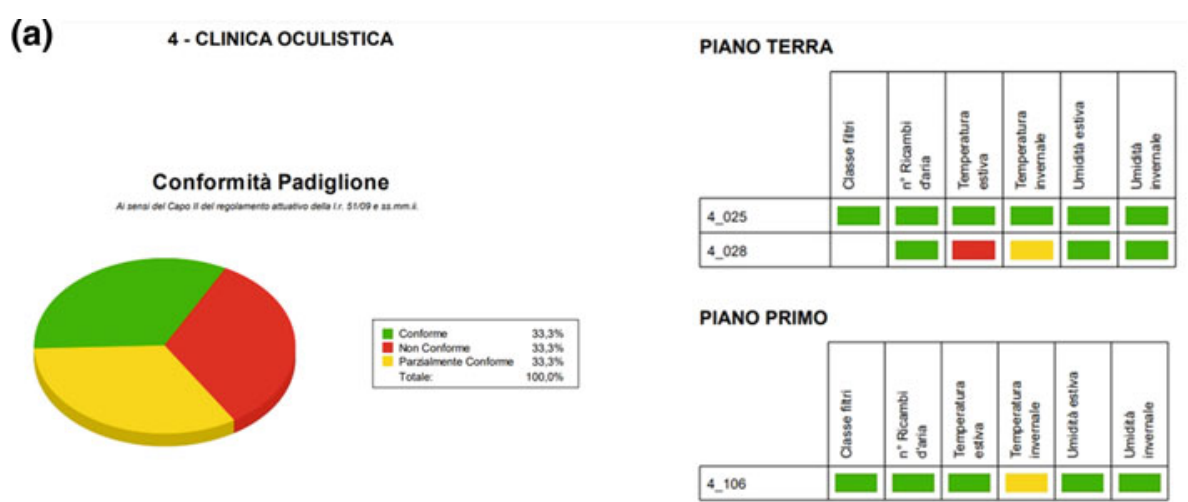

(b)

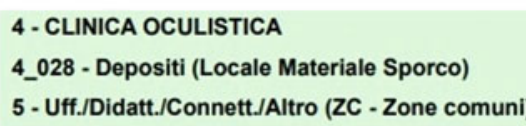

Temperatura estiva $\left[{ }^{\circ} \mathrm{C}\right]$

\section{Conclusion}

The proposed informative system has proven to be effective both for planning and verifying purposes. In fact, it can be used in development phases to design an efficient layout of energy saving according to the destination of use and activity area of different portions of the hospitals, as well as in a subsequent analysis, to verify the energy consumption in real-time or catch up, therefore allowing an effective intervention plan to improve efficiency wherever it would be needed.

\section{References}

1. Luschi, A., Miniati, R., Iadanza, E.: A Web based integrated healthcare facility management system. In: 6th European Conference of the International Federation for Medical and Biological Engineering. IFMBE Proceedings, vol. 45, pp. 633-636 (2015)

2. Iadanza, E., Marzi, L., Dori, F., Gentili, G.B., Torricelli, M.C.: Hospital health care offer. A monitoring multidisciplinar approach.
In: World Congress on Medical Physics and Biomedical Engineering 2006. IFMBE Proceedings, vol. 14, pp. 3685-3688 (2007)

3. Iadanza, E., Turillazzi, B., Terzaghi, F., et al.: The streamer European project. Case study: Careggi hospital in Florence. In: 6th European Conference of the International Federation for Medical and Biological Engineering. IFMBE Proceedings, vol. 45, pp. 649652 (2015)

4. Iadanza, E., Ottaviani, L., Guidi, G., Luschi, A., Terzaghi, F.: License: web application for monitoring and controlling hospitals' status with respect to legislative standards. In: XIII Mediterranean Conference on Medical and Biological Engineering and Computing 2013. IFMBE Proceedings, vol. 41, pp. 1887-1890 (2014)

5. Iadanza, E., Luschi, A., Ancora, A.: Bed management in hospital systems. In: World Congress on Medical Physics and Biomedical Engineering 2018. IFMBE Proceedings, vol. 68/3, pp. 313-316 (2019)

6. Luschi, A., Monti, M., Iadanza, E.: Assisted reproductive technology center design with quality function deployment approach. In: World Congress on Medical Physics and Biomedical Engineering, Toronto, Canada, 7-12 June 2015. IFMBE Proceedings, vol. 51, pp. 1587-1590 (2015)

7. Luschi, A., E., Marzi, L., Miniati, R., Iadanza, E.: A custom decision-support information system for structural and technological analysis in healthcare. In: XIII Mediterranean Conference on Medical and Biological Engineering and Computing 2013. IFMBE Proceedings, vol. 41, pp. 1350-1353 (2014) 